Acta Zoológica Mexicana (nueva serie), Volumen 38, 1-10.

https://doi.org/10.21829/azm.2022.3812454

Artículo original

\title{
Coccinélidos (Coleoptera: Coccinellidae) asociados a cítricos del centro-sur de Tamaulipas, México
}

\section{Coccinellids (Coleoptera: Coccinellidae) associated to citrus from the south-central of Tamaulipas, México}

\author{
${ }^{1}$ LuIS Alejandro LARA, ${ }^{2}$ SeRgio GodínEZ-CORTÉS, (iD ${ }^{1}$ Reyna \\ IVONNE TORRES, 'EDUARDO PADRON-TORRES, 'JOSÉ REYES, \\ (iD) ${ }^{1}$ FeLIPE ANASTACIO GONZÁLEZ-GONZÁLEZ, (iD) 1 VERóNICA \\ HERNÁNDEZ, 'EdUARdo DANIEl GONZÁlEZ-NIETO, \\ (iD 1*HERMELINDO HERNÁNDEZ
}

${ }^{1}$ Universidad Autónoma de Tamaulipas, Unidad Académica Multidisciplinaria Mante, Enrique Cárdenas González 1201 Pte., Col. Jardín, C. P. 89840, Ciudad Mante, Tamaulipas, México.

${ }^{2}$ Colegio de Postgraduados. Especialidad en Entomología y Acarología, Montecillos, Estado de México, Km 36.5 Carretera México-Texcoco, C. P. 562330, México.

Editor responsable: Alfredo Ramírez-Hernández

*Autor corresponsal:

iD Hermelindo Hernández hermelindo.torres@uat.edu.mx yemir12torres@gmail.com

Cita:

Lara, L. A., Godínez-Cortés, S.,

Torres, R. I., Padrón-Torres, E., Reyes, J., González-González, F. A., Hernández, V., González-Nieto, E.

D., Hernández, H. (2022)

Coccinélidos (Coleoptera: Coccinellidae) asociados a cítricos del centro-sur de Tamaulipas, México. Acta Zoológica Mexicana (nueva serie), 38, 1-10. 10.21829/azm.2022.3812454 elocation-id: e3812454

Recibido: 03 octubre 2021

Aceptado: 18 enero 2022

Publicado: 22 febrero 2022
RESUMEN. El presente estudio identifica e ilustra la riqueza de especies de Coccinellidae asociados a cultivos de cítricos en el centro-sur de Tamaulipas, México. La colecta de especímenes se realizó de marzo a octubre de 2020 en intervalos semanales, usando red entomológica, trampas amarillas y revisando manualmente las ramas de los árboles de menor tamaño. Se recolectaron 169 especímenes adultos de la familia Coccinellidae representados en dos subfamilias, 13 géneros y 20 especies. De las 20 especies, 14 ya estaban reportadas y seis son primeros registros para Tamaulipas. Se concluye que el aumento en el número de especies recolectadas en esta región se debe al mayor esfuerzo en el muestreo realizado.

Palabras clave: diversidad; especies; coleópteros; depredadores; nuevos registros

ABSTRACT. The present study identifies and illustrates the 
species richness of Coccinellidae associated with citrus crops in south-central Tamaulipas, Mexico. The collection of specimens was carried out from March to October 2020, at weekly intervals, using entomological netting, yellow traps and manually checking the branches of the smaller trees. A total of 169 adult specimens of the Coccinellidae family represented in two subfamilies, 13 genera and 20 species were collected. Of the 20 species, 14 were already reported and six are first records 2 for Tamaulipas. It is concluded that the increase in the number of species collected in this region is due to the more significant effort in the sampling carried out.

Key words: diversity; species; coleopters; predators; new records

\section{INTRODUCCIÓN}

Coccinellidae es una familia de insectos del orden Coleoptera que incluye aproximadamente 6,000 especies descritas, pertenecientes a 360 géneros y 42 tribus en el mundo (Nedvěd \& Kovár, 2012). Se conocen comúnmente como catarinitas y oscilan entre 0.8 y $18 \mathrm{~mm}$ de longitud (Seago et al., 2011). El 90 \% de las especies son depredadoras de áfidos (Hemiptera: Aphididae), cóccidos (Hemiptera: Pseudococcidae), psillidos (Hemiptera: Psyllidae), aleuródidos (Hemiptera: Aleyrodidae), larvas de crisomélidos (Coleoptea: Chrysomelidae) y ácaros (Acari: Tetranichidae), y el resto son fitófagos y micetófagos (Majerus, 1994; Hodek et al., 2012). Los coccinélidos son un grupo de coleópteros con importancia económica por su uso como agentes de control biológico y por su diversidad de adaptación a diferentes hábitats (Michels, 1987). Blackwelder (1944) reporta 44 géneros de esta familia y 180 especies para México. En el estado de Tamaulipas, RodríguezVélez et al. $(2016 ; 2019)$ identifican mediante métodos morfológicos y moleculares a 12 especies de coccinélidos asociados a Melanaphis sacchari Zehntner, 1897, en cultivos de sorgo; por su parte Ruiz-Cancino et al. (2006), menciona un listado de enemigos naturales de diferentes insectos considerados plagas para cultivos de cítricos, en los que incluye a siete especímenes y dos géneros no determinados a especie de coccinélidos.

La citricultura tiene una producción mundial superior a los 124 millones de toneladas, los principales países productores son China, Brasil, India, Estados Unidos de América, España y México (FAO, 2017). Actualmente, México cuenta con una superficie cultivada de 590,000 ha de cítricos (SIAP, 2019). Tamaulipas se ubica dentro de las entidades con mayor importancia en cuanto a producción de cítricos a nivel nacional, siendo la naranja dulce (Citrus sinensis L., 1753) y el limón (Citrus limon L., 1753) los de mayor producción, con aproximadamente 44,000 ha sembradas (SIAP, 2019). A pesar de la importancia que representa el cultivo de cítricos en el estado de Tamaulipas, se han realizado pocos estudios sobre la diversidad de especies presentes de la familia Coccinellidae. Por lo tanto, el objetivo de este trabajo fue identificar e ilustrar la riqueza de especies de coccinélidos asociados a cultivos de naranja dulce y limón persa de la región centrosur del estado de Tamaulipas, México.

\section{MATERIALES Y MÉTODOS}

La recolecta se llevó a cabo de marzo a octubre de 2020 en cultivos de naranja dulce C. sinensis y limón persa Citrus latifolia Tanaka, 1937, ubicados en el sur de Tamaulipas, específicamente en 
huertos del rancho "El Carmen", municipio de El Mante, entre los paralelos $22^{\circ} 44^{\prime} \mathrm{N}$ y $98^{\circ} 58^{\prime} \mathrm{O}$; en el municipio Nuevo Morelos localizado en $2^{\circ} 28^{\prime} 0^{\prime \prime} \mathrm{N}$ y a $0^{\circ} 46^{\prime} 42^{\prime \prime} \mathrm{O}$, y en el rancho "La Encantada", municipio El Naranjo, ubicado entre los límites del sur oeste del estado de Tamaulipas y San Luis Potosí, a $2^{\circ} 28^{\prime} 0^{\prime \prime} \mathrm{N}$ y $0^{\circ} 46^{\prime} 42^{\prime \prime} \mathrm{O}$.

Los coccinélidos se recolectaron cada ocho días con red entomológica y utilizando trampas amarillas con material adherente a base de grasa automotriz transparente, colocadas a media altura de los árboles de cítricos. También fueron revisadas manualmente las ramas de los árboles de menor tamaño.

Los insectos atraídos a las trampas se despegaban suavemente del plástico con pinceles sin deteriorarlos y se colocaban en frascos de plástico con alcohol al $80 \%$, al igual que el resto de ejemplares recolectados. Cada frasco fue etiquetado con la fecha y sitio de colecta para ser trasladados al Laboratorio de Botánica de la Unidad Académica Multidisciplinaria Mante de la Universidad Autónoma de Tamaulipas para su posterior identificación.

Para la determinación taxonómica se utilizaron los trabajos y claves dicotómicas de González, (2006), Perrier (1967), Plaza (1986), Raimundo (1986) y Smirnoff (1973). La confirmación de algunas especies (Chilochorus cacti, Coccinella septempunctata, Coleomegilla maculata, Cryptolaemnus montrouzier, Cycloneda sanguinea, Delphastus pusillus, Hippodamia convergens, Olla v-nigrum, Scymnus apicanus y Scymnus louisianae) fue realizada por el entomólogo Guillermo González F., especialista del grupo en Sudamérica.

Para la toma de fotografías y la observación de estructuras morfológicas internas y externas se utilizó una cámara Canon EOS Rebel t6 acoplada a un microscopio de disección Zeiss modelo EZ4E. Las imágenes se procesaron en el programa Zeiss Application Suite y fueron editadas con Adobe Photoshop CS5. El material se depositó en la colección entomológica del Colegio de Postgraduados campus Montecillos del Estado de México.

\section{RESULTADOS}

Se recolectaron 169 especímenes adultos de la familia Coccinellidae representados en dos subfamilias, 13 géneros y 20 especies (Figs. 1,2): 41especímenes recolectados en el rancho "El Carmen", 54 en huertos de Nuevo Morelos y 74 en el rancho "La Encantada". De las 20 especies, 14 ya estaban reportadas para el estado: Coccinella septempunctata Linnaeus, 1758, Coleomegilla maculata De Geer, 1758, Cycloneda sanguinea Linnaeus, 1763, Hippodamia convergens GuérinMéneville, 1842, Olla v-nigrum Mulsant, 1866, Psyllobora renifer Casey, 1899, Brachyacantha barberi Gordon, 1985, Brachyacantha decora Casey, 1899, Brachyacantha quadrillum, Le Conte, 1858, Scymnus horni Gorham, 1897, Scymnus loewii Mulsant, 1850, Scymnus louisianae J. Chapin, 1973, Delphastus pusillus Le Conte, 1852 y Chilochorus cacti Linnaeus, 1767 (Cuadro 1). Siendo seis primeros registros para la región centro-sur de Tamaulipas: Psyllobora bicongregata Boheman, 1859, Psyllobora confluents Fabricius, 1801, Cryptolaemnus montrouzieri Mulsant, 1850, Hyperaspis punctata Le Conte, 1880, Scymnus apicanus Chapin, 1973 y Stethorus punctum Le Conte, 1852 (Cuadro 1). 
Cuadro 1. Riqueza de especies de la familia Coccinellidae y su planta hospedera recolectados en huertos de cítricos en el centro-sur de Tamaulipas, México.

\begin{tabular}{|c|c|c|c|c|c|c|}
\hline \multirow{2}{*}{ Familia } & \multirow{2}{*}{ Subfamilia } & \multirow{2}{*}{ Nombre específico } & \multicolumn{3}{|c|}{$\begin{array}{c}\text { Abundancia de especies } \\
\text { por huertos }\end{array}$} & \multirow{2}{*}{$\begin{array}{c}\text { Planta hospedera: } \\
\text { Citrus sinensis y } \\
\text { Citrus latifolia }\end{array}$} \\
\hline & & & $\begin{array}{c}\text { El } \\
\text { Carmen }\end{array}$ & $\begin{array}{l}\text { Nuevo } \\
\text { Morelos }\end{array}$ & $\begin{array}{c}\text { La } \\
\text { Encantada }\end{array}$ & \\
\hline \multirow[t]{20}{*}{ Coccinellidae } & Coccinellinae & Chilocorus cacti L.* & & 2 & 15 & C. sinensis y C. latifolia \\
\hline & Latreille, 1807 & Cryptolaemus montrouzieri Muls. ** & & & 7 & C. sinensis y C. latifolia \\
\hline & & Coccinella septempunctata $\mathrm{L} . *$ & & 5 & 7 & C. sinensis y C. latifolia \\
\hline & & Coleomegilla maculata De G.* & & & 8 & C. sinensis y C. latifolia \\
\hline & & Cycloneda sanguinea $\mathrm{L}^{*}$ & 4 & 3 & 19 & C. sinensis y C. latifolia \\
\hline & & Hippodamia convergens G. M.* & 4 & 6 & 8 & C. sinensis y C. latifolia \\
\hline & & Olla v-nigrum Muls.* & 1 & 5 & 3 & C. sinensis y C. latifolia \\
\hline & & Psyllobora bicongregata Boh. ${ }^{* *}$ & 5 & & & C. sinensis \\
\hline & & Psyllobora confluens Fab.** & 8 & & & C. sinensis \\
\hline & & Psyllobora renifer C.* & & 6 & & C. sinensis y C. latifolia \\
\hline & & Brachyacantha barberi G.* & 4 & 3 & 5 & C. sinensis y C. latifolia \\
\hline & & Brachyacantha decora C.* & & 5 & 8 & C. sinensis y C. latifolia \\
\hline & & Brachiacantha quadrillum Le C.* & & 3 & & C. sinensis \\
\hline & & Hyperaspis punctata Le C.** & & 5 & & C. sinensis \\
\hline & & Scymnus apicanus J. C.** & & & 4 & C. sinensis \\
\hline & & Scymnus horni G.* & & 1 & 2 & C. sinensis \\
\hline & & Scymnus loewii Muls.* & 5 & 5 & & C. sinensis y C. latifolia \\
\hline & & Scymnus louisianae J. C.* & 4 & 1 & 3 & C. sinensis \\
\hline & & Stethorus punctum Le C.** & 6 & & & C. sinensis \\
\hline & $\begin{array}{l}\text { Microweiseinae } \\
\text { Leng, } 1920\end{array}$ & Delphastus pusillus Le C.* & & 4 & & C. sinensis \\
\hline Total & & & 41 & 54 & 74 & \\
\hline
\end{tabular}

**Primeros registros para la región centro-sur de Tamaulipas / *Especies ya registradas. C. sinensis: Naranja dulce; C. latifolia: Limón Persa. 

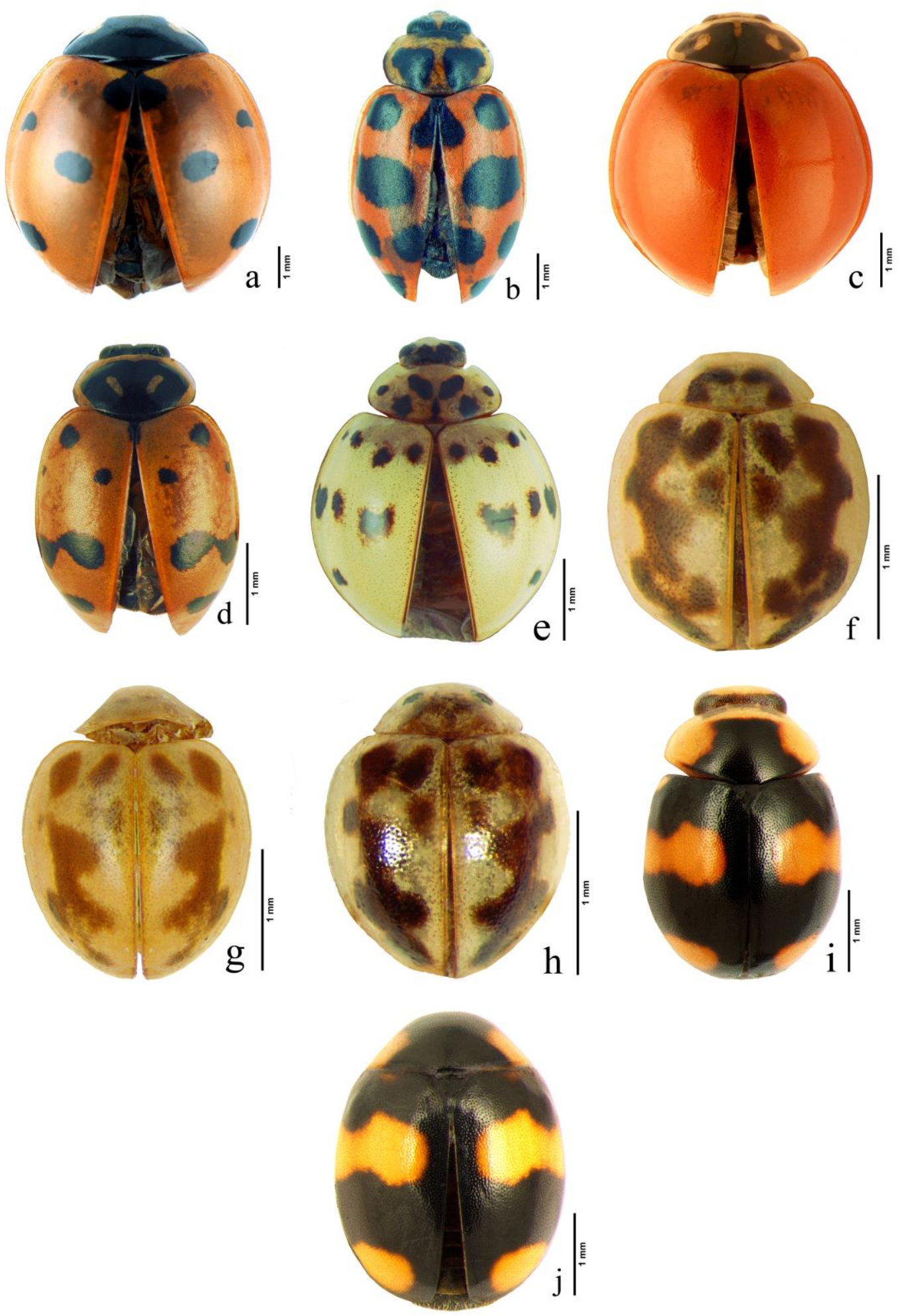

Figura 1. Especies de coccinélidos de la región centro-sur de Tamaulipas, México. Vista superior de los ejemplares adultos: a. Coccinella septempunctata L. b. Coleomegilla maculata De G. c. Cycloneda sanguinea L. d. Hippodamia convergens G. M. e. Olla v-nigrum Muls. f. Psyllobora bicongregata B. g. P. confluents Fab. h. P. renifer C. i. Brachyacantha barberi G. j. B. decora C. 

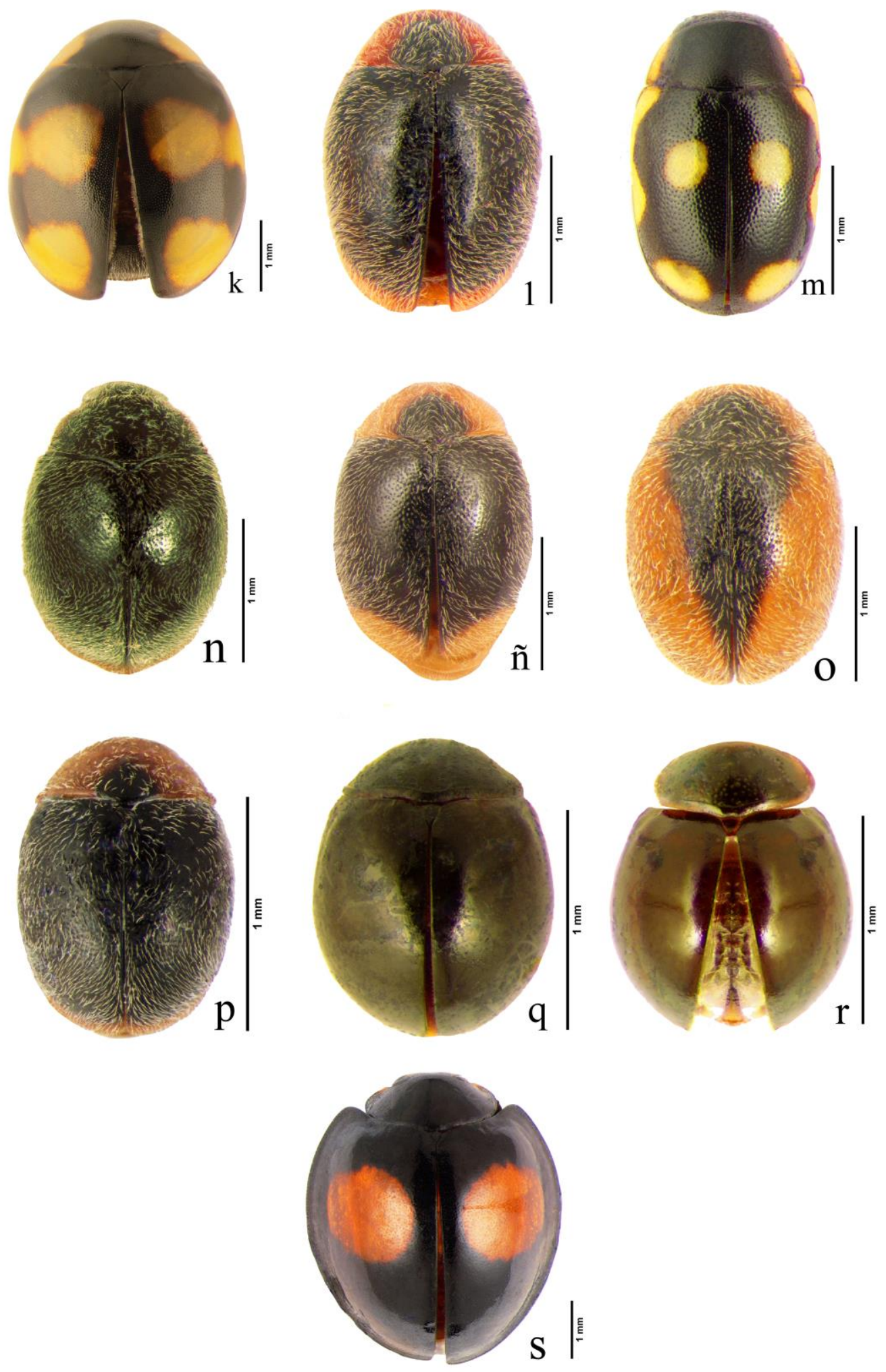

Figura 2. Especies de coccinélidos de la región centro-sur de Tamaulipas, México. Vista superior de los ejemplares adultos: k. B. quadrillum, Le C. I. Cryptolaemnus montrouzieri Muls. m. Hyperaspis punctata Le C. n. Scymnus apicanus J. C. ñ. S. horni G. o. S. loewii Muls. p. Scymnus Louisianae J. C. q. Stethorus punctum Le C. r. Delphastus pusillus Le C. s. Chilochorus cacti L. 
Material examinado: Coccinella septempunctata L: Colector: L. Alejandro Lara, 21-VI-2020, red entomológica. Huertos: Municipio Nuevo Morelos ( $2^{\circ} 28^{\prime} 0^{\prime \prime} \mathrm{N}, 0^{\circ} 46^{\prime} 42^{\prime \prime} \mathrm{O}$ ), 5 ejemplares; "La Encantada" (2० 28' 0" N, a $\left.0^{\circ} 46^{\prime} 42^{\prime \prime} \mathrm{O}\right) 7$ ejemplares. Coleomegilla maculata De G.: Colector: L. Alejandro Lara, 05-VII-2020, red entomológica. Huerto: "La Encantada" ( $2^{\circ} 28^{\prime} 0^{\prime \prime} \mathrm{N}, 0^{\circ} 46^{\prime} 42^{\prime \prime} \mathrm{O}$ ) 8 ejemplares. Cycloneda sanguinea L.: L. Alejandro Lara, 26-III-2020, 16-IV-2020, 05-V-2020, red entomológica y manual. Huertos: "El Carmen", Municipio El Mante (22 $\left.44^{\circ} \mathrm{N}, 98^{\circ} 58^{\prime} \mathrm{O}\right), 4$ ejemplares; Municipio Nuevo Morelos ( $2^{\circ} 28^{\prime} 0^{\prime \prime} \mathrm{N}, 0^{\circ} 46^{\prime} 42^{\prime \prime} \mathrm{O}$ ), 3 ejemplares; "La Encantada" (2० $\left.28^{\prime} 0^{\prime \prime} \mathrm{N}, 0^{\circ} 46^{\prime} 42^{\prime \prime} \mathrm{O}\right) 19$ ejemplares. Hippodamia convergens G-M.: Colector: L. Alejandro Lara, 16-VII-2020, 26-IX-2020, 02-V-2020, red entomológica. Huertos: "El Carmen", Municipio El Mante ( $\left.22^{\circ} 44^{\prime} \mathrm{N}, 98^{\circ} 58^{\prime} \mathrm{O}\right), 4$ ejemplares; Municipio Nuevo Morelos ( $\left.2^{\circ} 28^{\prime} 0^{\prime \prime} \mathrm{N}, 0^{\circ} 46^{\prime} 42^{\prime \prime} \mathrm{O}\right), 6$ ejemplares; "La Encantada" ( $\left.2^{\circ} 28^{\prime} 0^{\prime \prime} \mathrm{N}, 0^{\circ} 46^{\prime} 42^{\prime \prime} \mathrm{O}\right), 8$ ejemplares. Olla v-nigrum Muls.: Colector: L. Alejandro Lara, 02-V-2020, 21-VIII-2020, red entomológica. Huertos: "El Carmen", Municipio El Mante ( $\left.22^{\circ} 44^{\prime} \mathrm{N}, 98^{\circ} 58^{\prime} \mathrm{O}\right), 1$ ejemplar; Municipio Nuevo Morelos ( $\left.2^{\circ} 28^{\prime} 0^{\prime \prime} \mathrm{N}, 0^{\circ} 46^{\prime} 42^{\prime \prime} \mathrm{O}\right), 5$ ejemplares; "La Encantada" ( $\left.2^{\circ} 28^{\prime} 0^{\prime \prime} \mathrm{N}, 0^{\circ} 46^{\prime} 42^{\prime \prime} \mathrm{O}\right) 3$ ejemplares. Psyllobora renifer C.: Colector: L. Alejandro Lara, 06-V-2020, red entomológica. Huerto: Municipio Nuevo Morelos $\left(2^{\circ} 28^{\prime} 0^{\prime \prime} \mathrm{N}, 0^{\circ}\right.$ 46' 42" O), 6 ejemplares. Brachyacantha barberi G.: Colector: L. Alejandro Lara, 11-VIII-2020, 18-X2020, red entomológica y manual. Huertos: "El Carmen" Municipio El Mante ( $\left.22^{\circ} 44^{\prime} \mathrm{N}, 98^{\circ} 58^{\prime} \mathrm{O}\right)$, 4 ejemplares; Municipio Nuevo Morelos ( $\left.2^{\circ} 28^{\prime} 0^{\prime \prime} \mathrm{N}, 0^{\circ} 46^{\prime} 42^{\prime \prime} \mathrm{O}\right), 3$ ejemplares; "La Encantada" $\left(2^{\circ} 28^{\prime} 0^{\prime \prime} \mathrm{N}, 0^{\circ} 46^{\prime} 42^{\prime \prime}\right.$ O) 5 ejemplares. Brachyacantha decora C.: Colector: L. Alejandro Lara, 07IX-2020, red entomológica. Huertos: Municipio Nuevo Morelos ( $\left.2^{\circ} 28^{\prime} 0^{\prime \prime} \mathrm{N}, 0^{\circ} 46^{\prime} 42^{\prime \prime} \mathrm{O}\right), 5$ ejemplares; "La Encantada" ( $\left.2^{\circ} 28^{\prime} 0^{\prime \prime} \mathrm{N}, 0^{\circ} 46^{\prime} 42^{\prime \prime} \mathrm{O}\right) 8$ ejemplares. Brachyacantha quadrillum, Le C.: Colector: L. Alejandro Lara, 25-X-2020, red entomológica. Huerto: Municipio Nuevo Morelos ( $\left.2^{\circ} 28^{\prime} 0^{\prime \prime} \mathrm{N}, 0^{\circ} 46^{\prime} 42^{\prime \prime} \mathrm{O}\right), 3$ ejemplares. Scymnus horni G.: Colector: L. Alejandro Lara, 20-X-2020, red entomológica. Huertos: Municipio Nuevo Morelos ( $2^{\circ} 28^{\prime} 0^{\prime \prime} \mathrm{N}, 0^{\circ} 46^{\prime} 42^{\prime \prime} \mathrm{O}$ ), 1 ejemplar; "La Encantada" (2० 28' 0" N, $\left.0^{\circ} 46^{\prime} 42^{\prime \prime} \mathrm{O}\right) 2$ ejemplares. Scymnus loewii Muls.: Colector: L. Alejandro Lara, 28-IV-2020, red entomológica. Huertos: "El Carmen", Municipio El Mante $\left(22^{\circ} 44^{\prime} \mathrm{N}, 98^{\circ} 58^{\prime}\right.$ O), 5 ejemplares; Municipio Nuevo Morelos ( $2^{\circ} 28^{\prime} 0^{\prime \prime} \mathrm{N}, 0^{\circ} 46^{\prime} 42^{\prime \prime} \mathrm{O}$ ), 5 ejemplares. Scymnus louisianae J. C.: Colector: L. Alejandro Lara, 27-III-2020, 20-VII-2020, red entomológica. Huertos:

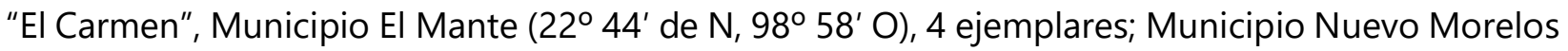
( $\left.2^{\circ} 28^{\prime} 0^{\prime \prime} \mathrm{N}, 0^{\circ} 46^{\prime} 42^{\prime \prime} \mathrm{O}\right), 1$ ejemplar; "La Encantada" ( $\left.2^{\circ} 28^{\prime} 0^{\prime \prime} \mathrm{N}, 0^{\circ} 46^{\prime} 42^{\prime \prime} \mathrm{O}\right), 3$ ejemplares. Delphastus pusillus Le C.: Colector: L. Alejandro Lara, 27-III-2020, red entomológica. Huerto: Municipio Nuevo Morelos ( $2^{\circ} 28^{\prime} 0^{\prime \prime} \mathrm{N}, 0^{\circ} 46^{\prime} 42^{\prime \prime} \mathrm{O}$ ), 4 ejemplares. Chilocorus cacti L.: Colector: L. Alejandro Lara, 07-IX-2020, 10-X-2020, red entomológica y manual. Huertos: Municipio Nuevo

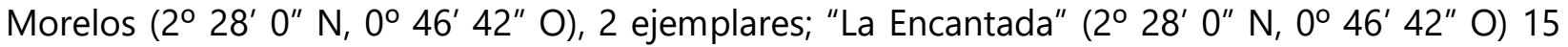
ejemplares. Psyllobora bicongregata Boh.: Colector: L. Alejandro Lara, 10-IV-2020, 23-V-2020, red

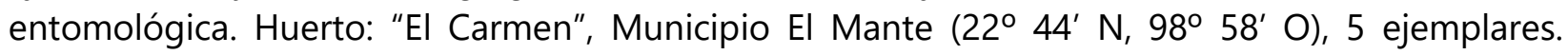
Psyllobora confluents Fab.: Colector: L. Alejandro Lara, 13-VI-2020, 20-VII,2020, red entomológica. Huerto: "El Carmen", Municipio El Mante (22 $\left.{ }^{\circ} 44^{\prime} \mathrm{N}, 98^{\circ} 58^{\prime} \mathrm{O}\right), 8$ ejemplares. Cryptolaemus montrouzieri Muls.: Colector: L. Alejandro Lara, 10-IV-2020, red entomológica. Huerto: "La Encantada" ( $\left.2^{\circ} 28^{\prime} 0^{\prime \prime} \mathrm{N}, 0^{\circ} 46^{\prime} 42^{\prime \prime} \mathrm{O}\right) 7$ ejemplares. Hyperaspis punctata Le C.: Colector: L. Alejandro Lara, 29-V-2020, red entomológica. Huerto: Municipio Nuevo Morelos ( $2^{\circ} 28^{\prime} 0^{\prime \prime} \mathrm{N}, 0^{\circ} 46^{\prime} 42^{\prime \prime} \mathrm{O}$ ), 5 ejemplares. Scymnus apicanus J. C.: Colector: L. Alejandro Lara, 11-VIII-2020, red entomológica. Huerto: "La Encantada" (2० 28' 0" N, 0 46' 42" O) 4 ejemplares. Stethorus punctum Le C.: Colector: 
Lara et al.: Coccinélidos asociados a cítricos

L. Alejandro Lara, 03-VIII-2020, red entomológica. Huerto: "El Carmen", Municipio El Mante (22 $\left.44^{\prime} \mathrm{N}, 98^{\circ} 58^{\prime} \mathrm{O}\right), 6$ ejemplares.

\section{DISCUSIÓN}

Los coccinélidos son depredadores de insectos herbívoros, tanto en la etapa adulta como en la etapa larvaria, por lo que se consideran importantes ecológica y económicamente por ser reguladores de plagas de cultivos agrícolas (Obrycki \& Kring, 1998). González (2010) menciona a C. cacti y a C. sanguinea como los depredadores más importantes de Lepidosaphes gloverii Packard, 1869 (Hemiptera: Diaspididae) y de Diaphorina citri Kugayama, 1908 (Hemiptera: Liviidae) en cultivos de cítricos en Cuba, mientras que Michaud (2004) refiere que $C$. sanguinea, Harmonia axidiris Pallas, 1773 y $O$. v-nigrum, son depredadores efectivos, específicamente de ninfas de $D$. citri. Gaona et al. (2000) mencionan la presencia de cuatro géneros de coccinélidos alimentándose de áfidos en el centro de Tamaulipas: Hippodamia Dejean, 1837, Cyclodema Dufour, 1863, Brachyacantha Dejean, 1837 y Stethorus Weise, 1885, mientras que Manzo-Martínez y Varela (1990) reportan a 12 especies y siete géneros en el centro de Tamaulipas: P. renifer, Hyperaspis connectens Thunberg, 1808, Hyperaspis levrati Mulsant, 1850, O. v-nigrum, Mulsantina luteodorsa Chapin, 1973, Brachyacantha subfasciata Mulsant, 1850, Brachyacantha dentipes Mulsant, 1850, B. quadrillum, Scymnus (Pullus) tenebricus Gordon, 1976, Scymunus (Pullus) marginicollis Mannerheim, 1843, Scymnus (Pullus) loweii Mulsant, 1850 y Arawana sp. Se reporta a P. confluents, $P$. bicongregata, $C$. montrouzieri, S. apicanus, $H$. punctata y S. punctum, como primeros registros del centro-sur de Tamaulipas.

En México, la información respecto a la gran diversidad de especies de Coccinellidae como enemigos naturales de áfidos, cóccidos y psilidos que afectan el desarrollo de cultivos de cítricos es escasa (Lomelí et al., 1998). Sin embargo, en el estado Tamaulipas la mayoría de los insectos plaga presentes en huertos citrícolas no causan grandes problemas, debido a que existe control natural por la acción de los enemigos naturales (Ruiz-Cancino, 1997) entre los cuales se encuentran los coccinélidos. Debido a esto, es necesario llevar a cabo estudios de distribución y ciclos biológicos que proporcionen mayor información y con esto conocer las especies presentes de coccinélidos en la región sur de Tamaulipas y su relación con las plagas agrícolas presentes.

Agradecimientos. A la Unidad Académica Multidisciplinaria Mante de la Universidad Autónoma de Tamaulipas, por el apoyo brindado para la realización de este trabajo.

\section{LITERATURA CITADA}

Blackwelder, R. E. (1944) Checklist of the coleopterous insects of México, Central America, The West Indies, and South America. Part 2. Smithsonian Institution United States National Museum, Bulletin 185, 189-341.

FAO (2017) Food and Agriculture Organization of the United Nation Crops. Disponible en: http://www.fao.org/brasil/noticias/detail-events/es/c/1040741/ (Consulta 02 de febrero de 2022). 
Gaona, G., Ruiz, E., Peña, R. (2000) Los pulgones (Homoptera: Aphididae) y sus enemigos naturales en naranja, Citrus sinensis (L.), en la zona centro de Tamaulipas, México. Acta Zoológica Mexicana (nueva serie), 81, 1-12. https://doi.org/10.21829/azm.2000.81811868

González, G. (2010) Actualización de la bibliografía y nuevos registros en Coccinellidae de América del Sur (Insecta: Coleoptera). Boletín de la Sociedad Entomológica Aragonesa, 47, 245-256.

González, G. (2006) Los Coccinellidae de Chile. Disponible en: http://www.coccinellidae.cl (última consulta 02 de febrero de 2020).

Hodek, I., Van Emden, H., Honek, A. (2012) Ecology and behaviour of the ladybird beetles (Coccinellidae). Blackwell Publishing Ltd., 560 pp.

Lomelí, F. J. R., Peña, M. R., Garduño, A. L. (1998) Posibilidades de control biológico de Toxoptera citricida (Kirkaldy) en México. Memorias del XXI Congreso Nacional de Control Biológico. Río Bravo, Tamaulipas, México, 31-32.

Majerus, M. E. N. (1994) Ladybirds. Harper Collins, London, 367 pp. https://doi.org/10.1017/S0007485300037317

Manzo, M. G., Varela, S. E. F. (1990) Coccinellidae (Coleoptera) en naranjo velencia Citrus cinensis (L.) en la zona centro de Tamaulipas. Memorias XXV. Congreso Nacional de Entomología. Oaxaca, Oaxaca, México, 185.

Michaud, J. P., Grand, A. K. (2004) The adaptive significance of sibling egg cannibalism in the Coccinellidae: Comparative evidence from three species. Annals of the Entomological Society of America, 97, 710-719. https://doi.org/10.1603/0013-8746(2004)097[0710:ASOSEC]2.0.CO;2

Michels, G. J. (1987) A checklist of the Coccinellidae (Coleoptera) of Wyoming. The Coleopterist Bulletin, 41, 249-255.

https://doi.org/10.1146/annurev.ento.43.1.295

Nedvěd, O., Kovář, I. (2012) Phylogeny and classification I. Pp. 1-12. En: I. Hodek, H. F. van Emden, A. Honěk (Eds.). Ecology and behavior of the ladybird beetles (Coccinellidae). República Checa, Honěk, Blackwell Publishing.

Obrycki, J. J., Kring, T. T. (1998) Predaceous Coccinellidae in biological control. Annual Reviews of Entomology, 58, 839-845.

https://doi.org/10.1146/annurev.ento.43.1.295

Perrier, R. (1967) La Faune de la France. Tome 5: Coleópteres. Libraire Delagrave. Pp. 159-166. En: Paris A.

Plaza, E. (1986) Clave para la identificación de los géneros y catálogo de las especies Españolas Peninsulares y Baleares de Coccinellidae. Graellsia, 19-45.

Raimundo, A., Alves, M. (1986) Revisão dos coccinelídeos de Portugal. Universidad de Evora, Portugal, $103 \mathrm{pp}$.

Rodríguez-Vélez, J. M., Rodríguez-Vélez, B., Sarmiento-Cordero, M. A., Palomares-Pérez, M., Arredondo-Bernal, H. C. (2016) Species of Coccinellidae (Coleoptera: Cucujoidea) associated with Melanaphis sacchari Zehntner (Hemiptera: Aphididae) in Tamaulipas, Mexico. Entomological news, 126, 97-105.

Rodríguez-Vélez, J. M., Gallou, A., Najar-Pacheco, M. A., Uribe-Mú, C. A., Huerta-Martínez, F. M., Contreras-Ramos, A., Arredondo-Bernal, H. C. (2019) Morphological and COI 
identification of predatory Coccinellidae (Coleoptera) of Melanaphis sacchari (Zehntner) (Hemiptera: Aphididae) of Mexico. The Coleopterist bulletin, 73 (1), 1-9.

Ruiz-Cancino, E., Coronado, B. J. M., Myartseva, S. N. (2006) Situación actual del manejo de plagas de los cítricos en Tamaulipas, México. Manejo Integrado de Plagas y Agroecología, Costa Rica, 78, 94-100. Disponible en: https://www.insectosdemexico.com/uploads/articulos/29.pdf (última consulta 02 de febrero de 2022).

Ruíz-Cancino, E. (1997) Control natural y biológico de plagas citrícolas en Tamaulipas, México. Memoria II Curso Internacional de Citricultura. Manejo Integral fitosanitario, 131-135.

Seago, A. E., Giorgi, J. A., Li, J., ślipiński, A. (2011) Phylogeny, classification and evolution of ladybird beetles (Coleoptera: Coccinellidae) based on simultaneous analysis of molecular and morphological data. Molecular Phylogenetics and Evolution, 60, 137-151. https://doi.org/10.1016/j.ympev.2011.03.015

SIAP (2019) Servicio de Información Agroalimentaria y Pesquera. Producción agrícola. México. Sagarpa. Disponible en: https://www.gob.mx/siap/acciones-y-programas/produccionagricola-33119 (última consulta 02 de febrero de 2022).

Smirnoff, W. A. (1973) Guía práctica para la identificación de las especies Palearticas del Género "Scymnus" (Coleóptera: Coccinellidae). Boletín de la estación central de Ecología, Quebec, Canadá, 2 (4), 51-61. 